\title{
Short- and long-term outcomes of laparoscopic organ-sparing resection for pancreatic neuroendocrine neoplasms
}

\section{Renchao Zhang ( $\square$ areenn@163.com )}

Zhejiang Provincial People's Hospital

Jun Ma

Zhejiang Provincial People's Hospital

Yi-Ping Mou

Zhejiang Provincial People's Hospital

Yu-Cheng Zhou

Zhejiang Provincial People's Hospital

Wei-Wei Jin

Zhejiang Provincial People's Hospital

Chao Lu

Zhejiang Provincial People's Hospital

\section{Research}

Keywords: Organ-sparing, Pancreatic neuroendocrine neoplasms, Laparoscopy, Pancreatectomy

Posted Date: April 6th, 2020

DOI: https://doi.org/10.21203/rs.3.rs-20636/v1

License: (1) This work is licensed under a Creative Commons Attribution 4.0 International License.

Read Full License

Version of Record: A version of this preprint was published at World Journal of Surgery on July 22nd, 2020. See the published version at https://doi.org/10.1007/s00268-020-05707-8. 


\section{Abstract}

\section{Background}

Pancreatic neuroendocrine neoplasms (PNENs) are rare neoplasm with long life expectancy. In this setting, patients may benefit from laparoscopic organ-sparing resection. There are few reports of laparoscopic organ-sparing resection for PNENs. The aim of this study was to evaluate the Short- and long-term outcomes of laparoscopic organ-sparing resection for PNENs.

\section{Methods}

A retrospective study was performed for patients with PNENs who underwent laparoscopic organ-sparing pancreatectomy between March 2005 and May 2018. The patients' demographic data, operative results, pathological reports, hospital courses, morbidity and mortality, and follow-up data (including pancreatic function, till August 2018) was analyzed.

\section{Results}

Thirty-five patients were identified. There were 9 male and 26 female patients, with a median age of 46 years (range, $25-75$ years). The mean BMI was $24.6 \pm 3.3 \mathrm{~kg} / \mathrm{m}^{2}$. Nine patients received laparoscopic enucleation, 20 received laparoscopic spleen-preserving distal pancreatectomy, and 6 received laparoscopic central pancreatectomy. The operative time, intraoperative blood loss, transfusion rate, postoperative hospital stay were $186.4 \pm 60.2 \mathrm{~min}, 165 \pm 73.0 \mathrm{ml}, 0,9 \mathrm{~d}$ (range, $5-23 \mathrm{~d}$ ), respectively. The morbidity rate, $\geq$ Grade $\otimes$ complication rate and $\geq$ grade $B$ pancreatic fistula rate were $34.2 \%, 11.4 \%, 8.7 \%$, respectively, with no mortality. The rate of follow-up was $94.3 \%$, and the median follow-up time was 55 months (range, 3-158months). One patients had diabetes after laparoscopic spleen-preserving distal pancreatectomy, no patient had symptom of pancreatic exocrine insufficiency. One patient developed recurrence 36 months after laparoscopic enucleation and was managed with surgical resection. The other patients survived without metastases or recurrence during the follow-up.

\section{Conclusions}

Laparoscopic organ-sparing resection for selected cases of PNENs is safe and feasible, and have favorable short- and long-term outcome.

\section{Background}

Pancreatic neuroendocrine neoplasms (PNENs) are rare neoplasms that represent about $2-4 \%$ of pancreatic tumors and have an incidence of 1 per 100,000 population[1,2]. The diagnosis of PNENs has increased over the past 40 years, most likely due to availability of high-resolution medical imaging and histopathological methods[3]. PNENs comprise a heterogeneous group of tumors with a very varied biological behavior. Complete surgical resection is the curative modality for resectable PNENs with the expectancy to long-term survival[3]. 
Organ-sparing pancreatectomy represents a limited resection alternative to pancreaticoduodenectomy (PD) and distal pancreatectomy (DP) for treatment of benign or low-grade malignant tumors of the pancreas[4-6]. This procedure is thought to be associated with minimal exocrine and/or endocrine insufficiency, better quality of life, and preservation of the spleen and its immunologic properties [46].Laparoscopic pancreatic surgery has been gaining popularity in the last decade due to recent technological developments in laparoscopic technique and instruments. So, laparoscopic organ-sparing pancreatectomy is thought to be an ideal procedure for the PNENs.

Previous study of laparoscopic pancreatectomy for PNENs has found advantages related to minimalaccess surgery and a good overall disease-specific long-term prognosis[7-9]. There are few reports of laparoscopic organ-sparing resection for PNENs[9,10]. The aim of this study was to evaluate the Shortand long-term outcomes of laparoscopic organ-sparing resection for PNENs.

\section{Methods}

Between March 2005 and May 2018, patients with PNENs who underwent laparoscopic organ-sparing pancreatectomy in our institution were reviewed. The preoperative assessment included a computed tomographic (CT) scan , a pancreatic magnetic resonance imaging (MRI), and an endoscopic ultrasound (EUS) or with fine-needle aspiration(FNA). The preoperative anesthesic evaluation was done using the American Society of Anesthesiology guidelines.

Preoperative, operative, and postoperative data were prospectively collected and retrospectively analyzed. The data included patient demographics, clinical presentation, intraoperative variables (type of resection, operative time, estimated blood loss (EBL), conversion to open operation, blood transfusion requirement), postoperative hospital stay, morbidity and mortality (within 30 days from surgery), readmission rate (within 30 days from hospital discharge), pathologic findings, and long-term follow-up.

Pancreatic fistula (PF) was assessed according to the International Study Group on Pancreatic Fistula recommendations [11]. Postoperative morbidity was graded using the Clavien-Dindo classification[12]. Graded $\nabla$ and $\nabla$ were grouped as minor and graded $\nabla-\otimes$ was considered as major complications [12].

Patients were followed up via out-patient examination. The final follow-up was taken in August 2018. Recurrence or distant metastasis was diagnosed pathologically by surgical resection, biopsy, or cytology and/or radiological examination. Pancreatic endocrine insufficiency was defined as new-onset diabetes and worsening diabetes[13]. Patients with diarrhea and steatorrhea, weight loss, and taking of pancreatic enzyme supplementation were considered to have pancreatic exocrine insufficiency[13].

The Institutional Review Board of Zhejiang provincial people's Hospital approved this study protocol.

\section{Operative technique}

All indications of operation were discussed by a multidisciplinary team board. The type of pancreatic resection was based on the locations and size of the tumors. Laparoscopic enucleation(LE) was 
performed for the tumors located $\geq 3 \mathrm{~mm}$ distant from the Wirsung duct (WD). Laparoscopic central pancreatectomy (LCP) was performed for the tumors located in the neck-body of the pancreas $<3 \mathrm{~mm}$ from the WD and which allowed $5 \mathrm{~cm}$ of the tail of the pancreas to be preserved. When the remaining tissue of the distal pancreas was fibrotic or atrophic or $<5 \mathrm{~cm}$ long, laparoscopic spleen-preserving distal pancreatectomy (LSPDP) was performed. LSPDP was performed for tumors located $<3 \mathrm{~cm}$ from the distal end of the pancreas and $<3 \mathrm{~mm}$ from the WD.

All of the operations were performed by one surgeon. The procedures were planned before surgery, but the final procedure was decided on during surgery based on a combination of macroscopic , intraoperative ultrasonographic findings, and intraoperative frozen section examination.

Patients were placed in supine position with the head slightly elevated. The surgeon and the second assistant who held the laparoscope stood on the right side of the patientand the first assistant stood on the left. Five trocars (three 5-mm trocars and two 10/12-mm trocars) are used; and the five trocars were arranged in a $V$ shape.

\section{Laparoscopic Enucleation,Laparoscopic Spleen-Preserving Distal Pancreatectomy, Laparoscopic Central Pancreatectomy}

The surgical technique for LE, LSPDP, and LCP have been previously described in detail elsewhere [1][1418].

\section{Histopathological data}

Histopathological data, such as size of the tumor, tumor location, margin status, lymph node status, were also analyzed. Tumors were reviewed and graded based on mitotic count and Ki-67 index, according to the World Health Organization(WHO) classification[19] and the European Neuroendocrine Tumor Society(ENETS) TNM classification[20].

\section{Statistical analysis}

Continuous clinicopathological data were expressed as mean \pm standard deviation or median (range) as appropriate. Categorical variables were reported as number and percentage. All statistical analyses were performed using SPSS version 19.0.

\section{Results}

From March 2005 to May 2018, a total of 35 patients with PNENs underwent laparoscopic organ-sparing pancreatectomy. Demographic variables of the 35 patients are given in Table 1. There were 9 male and 26 female patients, with a median age of 46 years (range, 25-75 years). The mean BMI was $24.6 \pm 3.3 \mathrm{~kg} / \mathrm{m} 2$. Four patients underwent previous laparotomy: two with cholecystectomy, one appendectomy, and one partial gastrectomy. Of the 35 patients, 10 cases $(28.6 \%)$ were functional tumors. All of them were insulinoma. The remaining 25 patients (71.4\%) were nonfunctional tumors. 
Various preoperative diagnostic tools were used. CT scanning was performed in 35 cases, localizing the tumor in 33 cases. MRI scanning was performed in 23 cases, localizing the tumor in 22 cases. EUS was performed in 15 cases, localizing the tumor in 15 cases. In 15 cases (42.8\%), pre-operative cytology was performed using FNA.

Nine tumor (25.7\%) was located in the pancreatic head and neck: 1 case has 2 lesions, $16(45.7 \%)$ in the body and $10(28.6 \%)$ in the tail of the pancreas. Nine LE (25.7\%), 6 LCP $(17.2 \%)$ and 20 LSPDP $(57.1 \%)$ were performed: 16 with Kimura technique and 4 Warshaw technique. Combined resections of adjacent organs were performed in four patients. A concomitant cholecystectomy was performed in three patients, a partial gastrectomy was performed in one patient. No patients converted to open surgery.

The postoperative data are given in Table 2. The mean operating time was $186.4 \pm 60.2 \mathrm{~min}$, and the mean EBL was $165 \pm 73.0 \mathrm{ml}$. The first flatus time, diet start time and postoperative hospital stay were $2.1 \pm 1.1 \mathrm{~d}$, $3 d($ range, $1-7 d), 9 d$ (range, 5-23d), respectively. There was no postoperative 30-day mortality.

Postoperative complications occurred in 12 patients (34.2\%). According to the Clavien-Dindo classification, most complications were classified as grade $\Downarrow$ or $\Downarrow$. The $\geq$ Grade $\Downarrow$ complications were occurred in 4 patients $(11.4 \%)$. Of the 35 patients, 3 patients $(8.7 \%)$ developed Grade B PF, no Grade C PF . Two patients required percutaneous drainage, 1 patient persisted peripancreatic drainage for 4 weeks. Two patients (5.8\%) developed hemorrhage. One patient was bleeding of splenic artery pseudoaneurysm after LSPDP(Kimura technique), and underwent splenic arterial embolization to hemostasis. Another patient was bleeding of gastroduodenal artery(GDA) after LCP, and underwent GDA embolization and laparoscopic evacuation of hematoma.

The histopathological data are given in Table 3. The mean tumor diameter was $2.2 \pm 1.5 \mathrm{~cm}$. According to the WHO classification[18], 24 patients(68.6\%) were graded $\mathrm{G} 1,11$ patients(31.4\%) G2, and no $\mathrm{G} 3$. According to ENETS TNM classification [19], 20 patients(57.1\%)were stage I, 13 patients(37.1\%) IIA, 2 patients(5.8\%) IIIA. ALL patients had an R0 status.

A total of 33 patients (follow-up rate $94.3 \%$ ) were followed up via out-patient examination. The median follow-up period was 55 months (range 3-158 months). One patient developed recurrence 36 months after LE and was managed with surgical resection. The other patients survived without metastases or recurrence during the follow-up. One patient experienced intestinal obstruction 2 years after LSPDP. One patient developed new-onset diabetes treated with oral drug after LSPDP. No patient had symptom of pancreatic exocrine insufficiency.

\section{Discussion}

Laparoscopic organ-sparing resection for PNENs was initially described by Gagner in 1996 [21].The patient underwent LE of an insulinoma of the anterior body of the pancreas in 3 hours, and the hospital stay was 4 days. No recurrence was seen in follow-up. Owing to complicated operation and low prevalence of PNENs, papers about laparoscopic organ-sparing resection for PNENs reported in the literature are limited[9,10]. Cienfuegos et al[9]documented the effectiveness of laparoscopic organ- 
sparing resection in selected cases of PNENs. However, there study also included 11 cases of Laparoscopic radical distal pancreatosplenectomy for PNENs[9]. Moekotte et al[10] performed 87 cases of LSPDP for PNENs with feasible short-term outcome. However, there study also included 142 cases of LSPDP for pathologies other than PNENs and without long-term outcome [10]. To the best of our knowledge, this study was just focused on laparoscopic organ-sparing resection for PNENs. Our study demonstrated that laparoscopic organ-sparing resection for selected cases of PNENs is safe and feasible, and have favorable short- and long-term outcome.

The morbidity rates of laparoscopic pancreatectomy for PNENs reported in literatures varied

greatly from $21.5 \%$ to $50 \%[8,9] . P F$ was the most frequent complication after laparoscopic pancreatectomy for PNENs. The PF rates varied greatly from $21.5 \%$ to $36.1 \%[8,9]$.These studies included both laparoscopic organ-sparing resection and standard resection. Cherif et al[22] reported that high rate of morbidity (76\%) and PF (grades B and C) (42\%) after open parenchyma-sparing resection for PNENs. The present study revealed that the postoperative complications occurred in 12 patients $(34.2 \%)$ and PF in 3 patients $(8.7 \%$, Grade B). Because the present study used the new version of International Study Group (ISGPS) definition and grading of postoperative pancreatic fistula[11]. Our pilot studies showed that the morbidity rates and PF rates of LE ,LSPDP and LCP were similar to open surgery[14,15,16,18]. Therefore, we believe that it is not the type of the techniques (Laparoscopic or Open), but the patient's condition and operation's difficulty level were associated with PF formation.

The methods of PD or DP, sacrificed the pancreatic parenchyma and spleen, leads to the endocrine and exocrine deficiency and possibility of overwhelming post-splenectomy infection (OPSI) and potential increased risk of malignancy. Hence, organ-sparing pancreatectomy has increasingly become an option to treat benign or low-grade malignant tumors in order to preserve pancreatic parenchyma and spleen as much as possible. Zhang et al[14ه15ه18]reported LE ,LSPDP and LCP are safe and feasible for benign or borderline malignant lesions with faster recovery. LCP is associated with better quality of life[18].Song et al[23]reported that $2(8 \%)$ and $10(38.5 \%)$ patients were associated with new-onset diabetes and weight loss, respectively. New-onset diabetes was less frequent after LCP than after Extended LDP (8\% vs. 30.8 $\%, p=0.037)$. Cienfuegos et al $(9)$ reported that none of the patients with early stages PNENs treated with laparoscopic organ-sparing techniques presented recurrence during mean follow-up of 51 months. In our study, only one patient developed recurrence 36 months after LE. The other patients survived without metastases or recurrence during the follow-up. Only one patient developed new-onset diabetes treated with oral drug after LSPDP. No patient had symptom of pancreatic exocrine insufficiency. So laparoscopic organ-sparing pancreatectomy for PNENs has advantages related to minimal-access and functionpreserving surgery.

Compared to standard resection, laparoscopic organ-sparing pancreatectomy was nonoptimal oncologic resection with limited margin and nonstandard lymphadenectomy. Cherif et al[22] regarded that parenchyma-sparing pancreatectomy should be considered for low grade tumor which was suspected in nonsecreting tumor below $2 \mathrm{~cm}$ in size or insulinoma in the absence of distant or lymph node metastasis 
on preoperative assessment. Cienfuegos et al[9] regarded that tumors with no vascular invasion (stages I (T1NOM0), IIA(T2NOMO) and IIB (T3NOMO) established by the ENETS) seem to be the best candidate for laparoscopic organ-sparing pancreatectomy. In our study, all of functional tumors were insulinoma. All of tumors were graded $\mathrm{G} 1$ or G2, no G3. And 20 patients(57.1\%)were stage I, 13 patients(37.1\%) IIA, 2 patients(5.8\%)IIIA. Most patients carry an overall excellent prognosis. So which part of the advanced stages of PNENs were suitable for laparoscopic organ-sparing pancreatectomy need more cases to evaluation.

In our study, the mean tumor diameter was $2.2 \pm 1.5 \mathrm{~cm}$. Most tumors were less than $2 \mathrm{~cm}$. The challenge was to locate the tumor and make the decision of surgical approach. The planned surgical approach is governed largely by the findings in preoperative localization studies, but may commonly change in accordance with intraoperative findings. During the operation, ultrasonic examination of the pancreas is used to locate neoplasms which were deep in the parenchyma and make sure the distance between neoplasm and the main pancreatic duct. If the neoplasms located deeply in the parenchyma or in the posterior aspect of the pancreatic head, a Kocher maneuver was performed.

The limitations of this study were its retrospective design with relatively small number of cases. The ideal study would be a large, prospective randomized trial, which was difficult to accomplish owing to the infrequent diagnosis of patients with PNENs that were suitable for laparoscopic organ-sparing pancreatectomy. Despite these limitations, we believe that this study could provide valuable evidence in clinical practice.

\section{Conclusions}

Laparoscopic organ-sparing resection for selected cases of PNENs is safe and feasible, and have favorable short- and long-term outcome.

\section{Abbreviations}

CT: Computed tomographic; DP: Distal pancreatectomy; EBL: estimated blood loss; ENETS :European Neuroendocrine Tumor Society; EUS: Endoscopic ultrasound ; FNA :fine-needle aspiration; LCP: Laparoscopic central pancreatectomy; LE: Laparoscopic enucleation; LSPDP :Laparoscopic spleenpreserving distal pancreatectomy; MRI: Magnetic resonance imaging; PD:Pancreatoduodenectomy; PF :Pancreatic fistula;PNENs: Pancreatic neuroendocrine neoplasms; WD :Wirsung duct; WHO: World Health Organization

\section{Declarations}

\section{Ethics approval and consent to participate}

The Institutional Review Board of each institute approved this study. The written informed consent was obtained from the patients before inclusion in the study. 


\section{Consent for publication}

All the authors express the consent for publication on World Journal of Surgical Oncology.

\section{Availability of data and materials}

The dataset supporting the conclusions of this article is included within the article.

\section{Competing interests}

Ren-Chao Zhang, Jun Ma, Yi-Ping Mou, Yu-Cheng Zhou, Wei-Wei Jin, and Chao Lu have no conflict of interest or financial ties to disclose.

\section{Funding}

There were no funding contributions to this study.

\section{Authors' contributions}

Zhang RC and Mou YP conceived and designed the study. Zhang RC, Mou YP and Zhou YC performed the operation. Ma J, Jin WW and Lu C collected and analyzed data. Zhang RC drafted the manuscript. Mou YP revised the manuscript. All authors read and approved the final manuscript.

\section{Acknowledgements}

Not applicable.

\section{Author details}

Department of Gastrointestinal \& Pancreatic Surgery, Zhejiang provincial people's Hospital, Key Laboratory of Gastroenterology of Zhejiang Province, People's Hospital of Hangzhou Medical College, 158 Shangtang Road, Hangzhou 310014, Zhejiang Province, China.

\section{References}

1. Halperin DM, Kulke MH, Yao JC. A tale of two tumors: treating pancreatic and extrapancreatic neuroendocrine tumors. Annu Rev Med 2015, 66:1-16.

2. Reid MD, Balci S, Saka B, et al. Neuroendocrine Tumors of the Pancreas: Current Concepts and Controversies. Endocr Pathol 2014, 25(1):65-79.

3. Dasari A, Shen C, Halperin D, et al. Trends in the Incidence, Prevalence, and Survival Outcomes in Patients With Neuroendocrine Tumors in the United States. Jama Oncol 2017, 3(10):1335-1342.

4. Aranha GV, Shoup M. Nonstandard pancreatic resections for unusual lesions. Am J Surg 2005, 189(2):223-228. 
5. Crippa S, Boninsegna L, Partelli S,et al. Parenchyma-sparing resections for pancreatic neoplasms. J Hepatobiliary Pancreat Sci 2010, 17(6):782-787.

6. Hackert T, Hinz U, Fritz S, et al.Enucleation in pancreatic surgery: indications, technique, and outcome compared to standard pancreatic resections. Langenbecks Arch Surg 2011, 396(8):1197-1203.

7. Al-Kurd A, Chapchay K, Grozinsky-Glasberg S, et al. Laparoscopic resection of pancreatic neuroendocrine tumors. World J Gastroenterol 2014, 20(17):4908-4916.

8. Haugvik SP, Marangos IP, Røsok BI, et al.Long-term outcome of laparoscopic surgery for pancreatic neuroendocrine tumors. World J Surg 2013, 37(3):582-590.

9. Cienfuegos JA, Salguero J, Núñez-Córdoba JM, et al. Short- and long-term outcomes of laparoscopic organ-sparing resection in pancreatic neuroendocrine tumors: a single-center experience. Surg Endosc 2017, 31(10):3847-3857.

10. Moekotte AL, Lof S, White SA, et al. Splenic preservation versus splenectomy in laparoscopic distal pancreatectomy: a propensityscore-matched study. Surg Endosc 2020, 34(3):1301-1309.

11. Bassi C, Marchegiani G, Dervenis C, et al. The 2016 update of the International Study Group (ISGPS) definition and grading of postoperative pancreatic fistula: 11 Years After. Surgery 2017, 161(3):584591.

12. Dindo D, Demartines N, Clavien PA. Classification of surgical complications: a new proposal with evaluation in a cohort of 6336 patients and results of a survey. Ann Surg 2004, 240(2):205-213.

13. Hirono S, Tani M, Kawai M, et al. A central pancreatectomy for benign or low-grade malignant neoplasms. J Gastrointest Surg 2009, 13(9):1659-1665.

14. Zhang RC, Zhou YC, Mou YP, et al. Laparoscopic versus open enucleation for pancreatic neoplasms: clinical outcomes and pancreatic function analysis. Surg Endosc 2016, 30(7):2657-2665.

15. Zhang RC, Yan JF, Xu XW, et al. Laparoscopic vs open distal pancreatectomy for solid pseudopapillary tumor of the pancreas. World J Gastroenterol 2013, 19(37):6272-6277.

16. Yan JF, Xu XW, Jin WW, et al.Laparoscopic spleen-preserving distal pancreatectomy for pancreatic neoplasms:A retrospective study. World J Gastroenterol 2014, 20(38): 13966-13972.

17. Zhang R, Xu X, Yan J, et al. Laparoscopic central pancreatectomy with pancreaticojejunostomy: preliminary experience with 8 cases. J Laparoendosc Adv Surg Tech A 2013, 23(11):912-918.

18. Zhang RC, Zhang B, Mou YP, et al. Comparison of clinical outcomes and quality of life between laparoscopic and open central pancreatectomy with pancreaticojejunostomy. Surg Endosc 2017,31(11):4756-4763.

19. Bosman FT: WHO classification of tumours of the digestive system; 2010.

20. Rindi G, Klöppel G, Alhman H, et al. TNM staging of foregut (neuro)endocrine tumors: a consensus proposal including a grading system. Virchows Arch 2006,449(4):395-401.

21. Gagner M, Pomp A, Herrera MF. Early experience with laparoscopic resections of islet cell tumors. Surgery 1996, 120(6):1051-1054. 
22. Cherif R, Gaujoux S, Couvelard A, et al. Parenchyma-Sparing Resections for Pancreatic Neuroendocrine Tumors. J Gastrointest Surg 2012, 16(11):2045-2055.

23. Song KB, Kim SC, Park KM, et al. Laparoscopic central pancreatectomy for benign or low-grade malignant lesions in the pancreatic neck and proximal body. Surg Endosc 2015, 29(4):937-946.

\section{Tables}

Table 1. Baseline characteristics

\begin{tabular}{lc}
\hline Age $($ year $)$ & 46 (range,25-75) \\
Gender $($ Male/Female) & $9 / 26$ \\
BMI $\left(\mathrm{kg} / \mathrm{m}^{2}\right)$ & $24.6 \pm 3.3$ \\
Diabetes preoperative & $1(2.9 \%)$ \\
ASA score & \\
1 & $8(22.9 \%)$ \\
2 & $27(77.1 \%)$ \\
Previous laparotomy & $4(11.4 \%)$ \\
Type of tumor & \\
Functional & $10(28.6 \%)$ \\
$\quad$ Insulinoma & $10(28.6 \%)$ \\
$\quad$ Non-functional & $25(71.4 \%)$ \\
Tumor location & \\
Head-neck & $9(25.7 \%)$ \\
Body & $16(45.7 \%)$ \\
Tail & $10(28.6 \%)$ \\
Type of surgery & \\
Enucleation & $9(25.7 \%)$ \\
Central pancreatectomy & $6(17.2 \%)$ \\
Spleen-preserving distal pancreatectomy & $20(57.1 \%)$ \\
\hline
\end{tabular}

Data are expressed as mean \pm SD or median (range) or $\mathrm{n}(\%)$.

SD,standard deviation; BMI, body mass index; ASA, American Society of Anesthesiologists.

Table 2. Surgical Outcomes 


\begin{tabular}{lc}
\hline Operative outcomes & \\
Operating time( min) & $186.4 \pm 60.2$ \\
EBL( ml ) & $165 \pm 73.0$ \\
Transfused patients & 0 \\
Postoperative outcomes & $2.1 \pm 1.1$ \\
First flatus time(days) & $3($ range, $1-7)$ \\
Diet start time(days) & $9($ range,5-23) \\
Postoperative hospital stay (days) & $12(34.2 \%)$ \\
Overall complications & $8(22.8 \%)$ \\
$\quad$ Minor complications (Grade 0,0$)$ & $4(11.4 \%)$ \\
$\quad$ Major complications ( $\geq$ Grade $\square)$ & $3(8.7 \%)$ \\
Pancreatic fistula & $3(8.7 \%)$ \\
$\quad$ Grade B & 0 \\
$\quad$ Grade C & $2(5.8 \%)$ \\
Bleeding & $1(2.9 \%)$ \\
Delayed gastric empty & $2(5.8 \%)$ \\
Intra-abdominal infection & $1(2.9 \%)$ \\
Reoperation & $2(5.8 \%)$ \\
Percutaneous drainage & 0 \\
Readmission & 0 \\
Mortality &
\end{tabular}

Data are expressed as mean \pm SD or median (range) or $\mathrm{n}(\%)$.

$E B L$, estimated blood loss.

Table 3. Pathologic characteristics

\begin{tabular}{lc}
\hline Neoplasm size on histopathology $(\mathrm{cm})$ & $2.2 \pm 1.5$ \\
WHO classification (2010) & \\
G1 & $24(68.6 \%)$ \\
G2 & $11(31.4 \%)$ \\
G3 & 0 \\
Tumor stage (TNM) & $20(57.1 \%)$ \\
I (T1N0M0) & $13(37.1 \%)$ \\
IIA(T2N0M0) & $2(5.8 \%)$ \\
IIIA (T4N0M0) & $35(100 \%)$ \\
\hline
\end{tabular}


Data are expressed as mean \pm SD or $\mathrm{n}(\%)$ or unless otherwise specified. World Health Organization,WHO. 